Coldharbour Lane, Bristol, UK: ${ }^{2}$ Department of Gastroenterology, Institute of Tanslational Medicine, Liverpool, UK

Introduction Rotavirus is the most common cause of severe diarrhoea among infants and young children. Rotavirus is usually an easily managed disease of childhood, but worldwide nearly 500000 children under 5 years of age still die from rotavirus infection each year and almost two million more become severely ill. Rotavirus A (responsible for about $90 \%$ of infections) is typically diagnosed by finding the virus in the child's stool by enzyme immunoassay. This study was undertaken to expand our knowledge of VOCs from the stool of children and assess whether rotavirus causes easily measurable changes in the gut chemistry of infected young children.

Methods The volatile organic compounds (VOCs) from the stool of 53 children from Malawi (26 non-infected children with an unspecified GI problem and 27 rotavirus children diagnosed with the virus) were analysed using Headspace Trap-GC/MS. The faecal samples were placed in headspace vials and were heated from frozen to $90^{\circ} \mathrm{C}$. The VOC's were preconcentrated and focused prior to GC/ $\mathrm{MS}$ analysis. Those VOCs were identified by comparing their mass spectra with those contained in the NIST/EPA/NIH Mass Spectral Database.

Results A total of 186 different compounds have so far been identified. Of the 53 stool samples ethanol was found in $69 \%$ and $63 \%$ samples respectively between the non and infected classes, which contrasts with previous work where ethanol was found in all healthy adult and all healthy neonate stool. This could be due to the more dilute stool due to diarrhoea. Carbon disulphide has previously been found to be ubiquitous in healthy adult stool, again the frequency was much less at $27 \%$ and $22 \%$ respectively. In contrast the majority of samples contained ethanoic acid, with more samples in the rotavirus group. There were very little differences in the frequency (ca. 92\%) and abundance of ethanol in both sample classes, curiously ethanol has been previously shown to be present in all adult samples and absent in neonates. In contrast 2,3-butanedione and other aldehydes were significantly present both at greater frequency and typically at higher concentrations in rotavirus samples compared to non-rota samples, see Abstract PWE-169 figure 1

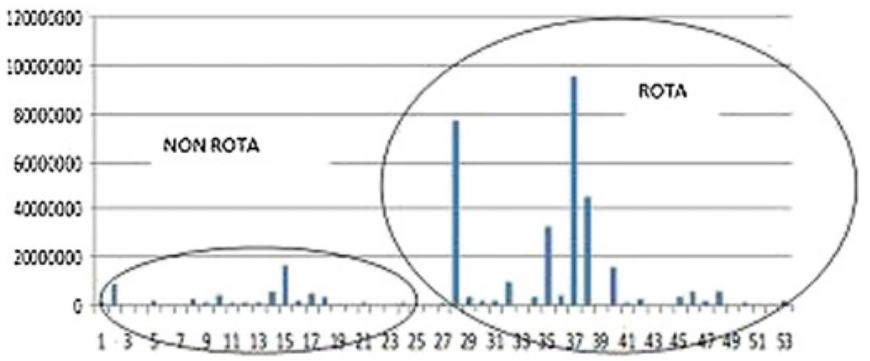

Abstract PWE-169 Figure 1 Combined peak area (arbitrary units) of selected carbonyl compounds (namely; 2,3-butanedione/propanal/2heptenal, (Z)-/octanal/nonanal combined) present in faecal samples analysed.

Conclusion Very little work has been published on volatile compounds from stool in particular from the stool of children, this work adds to this knowledge. Some compounds such as ethanol were found in approx. equal amounts in the diarrhoea of infected and non-infected stool, however in general there was a greater frequency and abundance of VOCs in the rota infected samples, particularly of aldehydes and 2,3-butandione.

Competing interests None declared

\section{PWE-170 PROTON PUMP INHIBITORS AND CLOPIDOGREL COMBINATION: IS THERE ANY RISK?}

doi:10.1136/gutjnl-2012-302514d.170

I Haq,* P Priyadharshan, S Lindsay, J Kurian, S Bulugahapitiya. Department of Cardiology, Bradford Hospitals, NHS Trust, Bradford, UK

Introduction Dual anti-platelet therapy (DAT) with aspirin and clopidogrel is prescribed up to 1 year following Acute Coronary Syndrome (ACS). Proton Pump Inhibitors (PPIs) are often used in selective patients to reduce the risk of gastrointestinal (GI) bleeding. Some studies have suggested that there is a possible interaction between PPIs and clopidogrel resulting in an adverse cardiac event rate. Main objective of this study was to determine if prescription of PPIs in ACS patients on dual anti-platelet therapy results in increased cardiac events compared to patients not receiving PPIs.

Methods A retrospective observational, comparative study of 200 admissions with ST elevation myocardial infarctions (STEMIs) and non-ST elevation myocardial infarction (nSTEMIs) over a period of 1 year. Patients were divided in two groups: PPI group (Patients on DAT and PPIs) and Non-PPI group (Patients on DAT only). End points were readmissions with ACS or death

Results Mean age was 67 (24-96), 65\% male and 35\% female, 75\% Caucasian and 25\% Asians. 27 patients were excluded (24 patients not receiving DAT, 3 patients on $\mathrm{H} 2$ antagonist). For PPI group (54\% on lansoprazole, $35 \%$ on pantoprazole and $11 \%$ on omeprazole), baseline characteristics were (Patient number-89, 27-STEMIs, 62nSTEMIs; mean age-69) whereas the corresponding values for nonPPI group were (Patient number-84, 38-STEMIs, 46-nSTEMIs, mean age-63). Mean GRACE score at admission (predictor of death or MI in 6 months) was $29 \%$ for PPI group whereas the corresponding value for the non-PPI group was $27 \%(p=0.075) .26$ patients were re-admitted with ACS, 20 in PPI group and six in nonPPI group (OR 3.76, 95\% CI 1.43 to 9.92, z statistic $2.68, p=0.007$ ). Five deaths due to ACS were observed, four in PPI group and one in non-PPI group (RR 3.77, 95\% CI 0.43 to 33.09 , $\mathrm{p}=0.23$ ).

Conclusion This study suggests that there is an association between PPIs and clopidogrel resulting in increased readmission with acute coronary syndromes but not in increased mortality. Larger scale studies are required to confirm or refute these observations.

Competing interests None declared.

\section{PWE-171 STRUCTURE-FUNCTION ANALYSIS OF POLYMORPHIC VACA TOXIN VARIANTS FROM HELICOBACTER PYLORI USING A RECOMBINANT APPROACH}

doi:10.1136/gutjnl-2012-302514d.171

1,2 J Winter, ${ }^{* 1,2} \mathrm{D}$ Letley, ${ }^{1,2} \mathrm{~K}$ Robinson, ${ }^{1,2} \mathrm{~J}$ Atherton. ${ }^{1}$ School of Clinical Sciences, The University of Nottingham, Nottingham, UK; ${ }^{2}$ Nottingham Digestive Diseases Centre Biomedical Research Unit, The University of Nottingham, Nottingham, UK

Introduction The Gram negative bacterium Helicobacter pylori persistently colonises the stomachs of around half the world's population. It causes chronic asymptomatic gastritis, and leads to peptic ulceration and gastric cancer in a small proportion of cases. One of the key factors influencing the development of more severe pathology and disease is the expression of virulence factors by the colonising strain. The vacuolating cytotoxin, VacA, is a major determinant of $H$ pylori pathogenicity and is highly polymorphic. Strains producing more active forms of VacA (i1 types) are more strongly associated with gastric cancer than strains producing less toxigenic VacA (i2 types). ${ }^{1}$ The i-region polymorphism is characterised by 10 amino acid substitutions and a serine-asparagineglutamine (SNQ) tri-peptide insertion in i2. We aimed to further characterise the structure and function of this important region. 